\title{
Local continuity laws on the phase space of Einstein equations with sources
}

\author{
R. Cartas-Fuentevilla \\ Instituto de Física, Universidad Autónoma de Puebla, Apartado Postal J-48 7250 Puebla, Pue., \\ México, and Enrico Fermi Institute, University of Chicago, 5640 S. Ellis Ave., Chicago, Illinois \\ 60637
}

Local continuity equations involving background fields and variations of the fields, are obtained for a restricted class of solutions of the Einstein-Maxwell and Einstein-Weyl theories using a new approach based on the concept of the adjoint of a differential operator. Such covariant conservation laws are generated by means of decoupled equations and their adjoints in such a way that the corresponding covariantly conserved currents possess some gauge invariant properties and are expressed in terms of Debye potentials. These continuity laws lead to both a covariant description of bilinear forms on the phase space and the existence of conserved quantities. Differences and similarities with other approaches, and extensions of our results are discussed.

PACS numbers: 04.20.Jb, 04.40.Nr

Running title: Local continuity laws....

\section{INTRODUCTION}

The study of the conservation laws is present in practically all areas of physics. In the context of the theories involving gravity, such a study becomes particularly interesting because of the lack of conserved currents representing the conservation of energy and momentum. Moreover, in the covariant description of a symplectic structure on the phase space of classical systems, a covariantly conserved two-form on such phase space is required. Such descriptions yield a canonical formalism manifestly covariant in field theory, which represents a starting point for a quantum mechanical treatment [1]. More specifically, in this context, the phase space is defined as the manifold of solutions of the classical equations of motion. Such a definition has the advantage of manifest covariance [1]. The existence of a two-form (say $\left.J^{\mu}\right)$, covariantly conserved $\left(\partial_{\mu} J^{\mu}=0\right)$ on such phase space, lead to a symplectic structure $\omega$ defined as $\omega \equiv \int_{\Sigma} J^{\mu} d \Sigma_{\mu}$ (where $\Sigma$ is an initial value hypersurface), 
independent of the choice of $\Sigma$. Moreover, the condition that Poisson brackets satisfy the Jacoby identity, holds if $\omega$ is closed (which means that $\omega$ is a two-form whose exterior derivative vanishes). This condition is satisfied if $J^{\mu}$ itself is closed. Several methods have been proposed in the literature for constructing these conserved currents, from the guess for the explicit form of the currents [1], to the very general result on the existence of a conserved current (the symplectic current) for the field variations for any field theory derived from a Lagrangian action [1, 2]. Alternatively, equivalent conserved currents have been obtained directly from the equations for the field variations using the fact that there exists a conserved current associated with any system of homogeneous linear partial differential equations that can be written in terms of a self-adjoint operator [3, 何. Such results have been obtained without any statement about the background fields. Nevertheless, the currents constructed in these ways are associated with the variations of the gauge fields. For example, in the case of the Einstein-Maxwell theory, such currents are expressed in terms of the metric and vector potential variations, and in this manner are defined modulo gauge transformations [1-4]. However, as it will be shown in the present paper, one can construct, for a restricted class of spacetimes, conserved currents (and the geometrical structures associated) without the gauge freedom of the matter fields. The approach is based on the concept of the adjoint of a differential operator, which represents an extension of that employed in Refs. [3, 何. Our starting point is the existence of appropriate decoupled equations for the restricted class of spacetimes under study, which have been obtained using the Newman-Penrose formulation [5. 6. It is shown that the conserved currents are also independent of the perturbed tetrad gauge freedom, which appears when the variations of the Newman-Penrose quantities are involved.

A previous application of the present approach has been given in the study of the perturbations of the Schwarzschild black hole, leading up to an important result on the existence of conservations laws for non-Hermitian systems, which has a direct extension to the scheme of string theory [7]. In this manner, another of the purposes of this article is to establish conservation laws for more general spacetimes, analogous to that appearing in Ref. [7]. However, unlike the results given in that reference, one of the main novelties in the present article is the interpretation of the corresponding conserved currents as bilinear forms on the phase space, which is connected with the very important concept of symplectic structures. This subject will be extended later on. 
The outline of this paper is as follows. Section II is dedicated to establish the general relationship between adjoint operators and conserved currents. The application of this very general result for identifying the conserved currents for the field variations in the Einstein-Maxwell and Einstein-Weyl theories is given in Sec. III. The properties of such conserved currents, and their relations with the existence of symplectic structures on the phase space are discussed. We finish in Sec. IV with some concluding remarks and future extensions of the present results.

\section{ADJOINT OPERATORS AND CONSERVED CURRENTS}

New branch: In Refs. [3, 田 it has been shown that there exists a conserved current associated with any system of homogeneous linear partial differential equations that can be written in terms of a self-adjoint operator. This result is limited for a self-adjoint system, for which the corresponding conserved current depends on a pair of solutions admitted by such a system. However, as we shall see below, there exists a more general possibility that extends for systems of equations which are not self-adjoint necessarily. The demonstration is very easy, following the basic idea of Refs. [3, 4]:

In accordance with Wald's definition [7], if $\mathcal{E}$ corresponds to a linear partial differential operator which maps $m$-index tensor fields into $n$-index tensor fields, then, the adjoint operator of $\mathcal{E}$, denoted by $\mathcal{E}^{\dagger}$, is that linear partial differential operator mapping $n$-index tensor fields into $m$-index tensor fields such that

$$
g^{\rho \sigma \ldots}\left[\mathcal{E}\left(f_{\mu \nu \ldots}\right)\right]_{\rho \sigma \ldots}-\left[\mathcal{E}^{\dagger}\left(g^{\rho \sigma \ldots}\right)\right]^{\mu \nu \ldots} f_{\mu \nu \ldots}=\nabla_{\mu} J^{\mu},
$$

where $J^{\mu}$ is some vector field depending on the fields $f$ and $g$. From Eq. (1) we can see that this definition automatically guarantees that, if the field $f$ is a solution of the linear system $\mathcal{E}(f)=0$ and $g$ a solution of the adjoint system $\mathcal{E}^{\dagger}(g)=0$, then we obtain the local continuity law $\nabla_{\mu} J^{\mu}=0$, which establishes that $J^{\mu}$ is a covariantly conserved current. This fact means that for any homogeneous equation system, one can always construct a conserved current taking into account the adjoint system. This general result contains the self-adjoint case $\left(\mathcal{E}=\mathcal{E}^{\dagger}\right)$ as a particular one, for which $f$ and $g$ correspond to two independent solutions.

In the present work, $f$ and $g$ will be associated with the first-order variations of the backgrounds 
fields. Such field variations will correspond, on the phase space, to one-forms [1]. In this manner, the left-hand side of Eq. (1) can be understood as a wedge product on such phase space: $g \wedge \mathcal{E}(f)-$ $\mathcal{E}^{\dagger}(g) \wedge f=\nabla_{\mu} J^{\mu}$, and something similar for the bilinear form $J^{\mu}$ in its dependence on the fields $f$ and $g$ (the operators $\mathcal{E}, \mathcal{E}^{\dagger}$, and $\nabla_{\mu}$ are depending only on the background fields).

Traditional branch: It is important, for completeness, that we outline the original idea for introducing the definition (1) in Ref. []: reduction of systems of linear partial differential equations to equations for scalar potentials (called Debye potentials), which determine a complete solution of the original system.

If we have the linear system $\mathcal{E}(f)=0$, and there exist linear operators such that

$$
\mathcal{S E}=\mathcal{O} \mathcal{T}
$$

identically, then the field $\mathcal{S}^{\dagger}(\psi)$ satisfies the equation

$$
\mathcal{E}^{\dagger}\left(\mathcal{S}^{\dagger}(\psi)\right)=0
$$

provided that the scalar field $\psi$ satisfies

$$
\mathcal{O}^{\dagger}(\psi)=0
$$

In particular, if $\mathcal{E}$ is self-adjoint $\left(\mathcal{E}^{\dagger}=\mathcal{E}\right)$, then $f=\mathcal{S}^{\dagger}(\psi)$ is a solution of $\mathcal{E}(f)=0$. For example, in the case considered in the present work, the (matrix) operator governing the field perturbations in the Einstein-Maxwell and Einstein-Weyl theories are, in fact, self-adjoint [3, 国.

Moreover, the existence of operators $\mathcal{S}, \mathcal{O}$, and $\mathcal{T}$ satisfying the above identity, is equivalent to the existence of a decoupled system

$$
\mathcal{O}(\Psi)=0
$$

obtained from the original system $\mathcal{E}(f)=0$, such that the scalar field $\Psi=\mathcal{T}(f)$.

Now, we can mix both branches of the adjoint operators scheme: since the fields $\psi$ and $\Psi$ satisfy equation which are adjoints to each other, we can establish, in according to the first branch, that

$$
\psi(\mathcal{O} \Psi)-\left(\mathcal{O}^{\dagger} \psi\right) \Psi=\nabla_{\mu} \mathcal{J}^{\mu}(\psi, \Psi)
$$

which means that $\nabla_{\mu} \mathcal{J}^{\mu}(\psi, \Psi)=0$. Furthermore, since $\Psi$ is finally depending on $\psi\left(\Psi=\mathcal{T}(f)=\mathcal{T}\left(\mathcal{S}^{\dagger}(\psi)\right)\right)$, $\mathcal{J}^{\mu}$ is dependent only on $\psi$ (however, see the paragraph before Eq. (8)). 
On the other hand, although this result has been established assuming only tensor fields and the presence of a single equation, such a result can be extended in a direct way to equations involving spinor fields, matrix fields, and the presence of more than one field [8].

It is worth pointing out some issues on the notation. The first-order field variations appearing in Ref. $[5,6]$ are denoted by a superscript B. On the other hand, the field variations coincide, in according to Witten's interpretation [1], with an infinite-dimensional generalization of the usual exterior derivative, which is traditionally represented by the symbol $\delta$. However, in Ref. $[5,6]$, the Newman-Penrose formalism is used, in which the symbol $\delta$ is employed for denoting one of the directional derivatives defined by the null tetrad. In this manner, for avoiding confusion, we will maintain the symbol $\delta$ as usual in the Newman-Penrose notation, and the superscript B for the first-order field variations (the exterior derivative of background fields). In the present article, the exterior derivative will not be performed explicity, and it will be sufficient for our purposes to understand any quantity with the superscript B as a one-form on the phase space. Quantities without such a superscript will correspond to background fields, which mean zero-forms on the phase space [1]. With these previous considerations, formulae and notation of Ref. $[5,6]$ will be used throughout this paper.

\section{COVARIANT CONTINUITY LAWS}

In Ref. [5], the metric and the vector potential variations of the Einstein-Maxwell equations have been obtained starting from a decoupled set of equations governing certain combinations of field variations, and have the form:

$$
\mathcal{O}\left(\Psi^{\mathrm{B}}\right)=0,
$$

where the $4 \times 4$ matrix operator $\mathcal{O}$ can be read from Eq. $(24)$ of that reference, and $\left(\Psi^{\mathrm{B}}\right)$ is the matrix:

$$
\left(\Psi^{\mathrm{B}}\right)=\left(\begin{array}{c}
\Psi_{0}^{\mathrm{B}} \\
-\tilde{\Psi}_{1}^{\mathrm{B}} \\
\phi_{1} \tilde{\kappa}^{\mathrm{B}} \\
-\phi_{1} \tilde{\sigma}^{\mathrm{B}}
\end{array}\right),
$$


where

$$
\begin{aligned}
\tilde{\Psi}_{1}^{\mathrm{B}} & \equiv\left(2 \phi_{1} \Psi_{1}^{\mathrm{B}}-3 \Psi_{2} \phi_{0}^{\mathrm{B}}\right) / 2 \phi_{1}, \\
\tilde{\kappa}^{\mathrm{B}} & \equiv \kappa^{\mathrm{B}}+(D-2 \epsilon-\rho)\left(\phi_{0}^{\mathrm{B}} / 2 \phi_{1}\right), \\
\tilde{\sigma}^{\mathrm{B}} & \equiv \sigma^{\mathrm{B}}+(\delta-2 \beta-\tau)\left(\phi_{0}^{\mathrm{B}} / 2 \phi_{1}\right) .
\end{aligned}
$$

The decoupled system (2) was obtained provided that the background electromagnetic field is nonnull with one of its principal null directions being geodetic and shear free, which means that $\Psi_{0}=$ $0=\Psi_{1}, \kappa=0=\sigma$, and $\phi_{0}=0$ in the background [5]. Note that the variations of these vanishing background quantities are those involved in $\left(\Psi^{\mathrm{B}}\right)$. As described in previous Section, the variations of background fields correspond to one-forms, in particular, those ones in Eq. (4). However, as shown in Appendix B, they correspond to exact one-forms. This property will be useful below.

On the other hand, according to Wald's method used in Ref. [5], the matrix potential $(\psi)$, which determines the complete variations, satisfies the equations (see Eqs. (25) and (27) of Ref. [5])

$$
\mathcal{O}^{\dagger}(\psi)=0
$$

where

$$
(\psi)=\left(\begin{array}{c}
M_{1^{\prime}} \\
-M_{0^{\prime}} \\
\psi_{\mathrm{G}} \\
-\psi_{\mathrm{E}}
\end{array}\right) .
$$

Thus, from the definition (1) we have that $(\psi) \wedge \mathcal{O}\left(\Psi^{\mathrm{B}}\right)-\mathcal{O}^{\dagger}(\psi) \wedge\left(\Psi^{\mathrm{B}}\right)=\nabla_{\mu} J^{\mu}\left(\Psi^{\mathrm{B}}, \psi\right)$. The lefthand side contains terms of the form $M_{1^{\prime}} \wedge \mathcal{O}_{11}\left(\Psi_{0}^{\mathrm{B}}\right)-\mathcal{O}_{11}^{\dagger}\left(M_{1^{\prime}}\right) \wedge \Psi_{0}^{\mathrm{B}}$, where $\mathcal{O}_{11} \equiv \bar{\delta}-4 \alpha+\pi$ and $\mathcal{O}_{11}^{\dagger} \equiv(\bar{\delta}-4 \alpha+\pi)^{\dagger}=\bar{\delta}+3 \alpha+\bar{\beta}-\bar{\tau}$, are the entries 11 of the $4 \times 4$ matrices $\mathcal{O}$ and $\mathcal{O}^{\dagger}$ respectively (see Eqs. (24) and (25) in Ref. [5]). Taking into account that $\bar{\delta} \equiv \bar{m}^{\mu} \partial_{\mu}$, and that is acting on scalar fields, such a term can be expressed in the form $M_{1^{\prime}} \wedge \mathcal{O}_{11}\left(\Psi_{0}^{\mathrm{B}}\right)-\mathcal{O}_{11}^{\dagger}\left(M_{1^{\prime}}\right) \wedge \Psi_{0}^{\mathrm{B}}=\nabla_{\mu}\left(\bar{m}^{\mu} M_{1^{\prime}} \wedge \Psi_{0}^{\mathrm{B}}\right)$, and similarly for the remaining terms considering that the tetrad vectors $D, \Delta$, and $\delta$ are defined by $l^{\mu} \partial_{\mu}, n^{\mu} \partial_{\mu}$, and $m^{\mu} \partial_{\mu}$ respectively. In this manner, from Eqs. (2) and (5) we obtain explicity the following covariant conservation law:

$$
\begin{aligned}
& \nabla_{\mu} J^{\mu}=0, \quad J^{\mu} \equiv-l^{\mu}\left(M_{1^{\prime}} \wedge \tilde{\Psi}_{1}^{\mathrm{B}}+2 \phi_{1} \psi_{\mathrm{G}} \wedge \tilde{\sigma}^{\mathrm{B}}\right)-n^{\mu}\left(M_{0^{\prime}} \wedge \Psi_{0}^{\mathrm{B}}+2 \phi_{1} \psi_{\mathrm{E}} \wedge \tilde{\kappa}^{\mathrm{B}}\right) \\
&+m^{\mu}\left(M_{0^{\prime}} \wedge \tilde{\Psi}_{1}^{\mathrm{B}}+2 \phi_{1} \psi_{\mathrm{G}} \wedge \tilde{\kappa}^{\mathrm{B}}\right)+\bar{m}^{\mu}\left(M_{1^{\prime}} \wedge \Psi_{0}^{\mathrm{B}}+\phi_{1} \psi_{\mathrm{E}} \wedge \tilde{\sigma}^{\mathrm{B}}\right) .
\end{aligned}
$$


It is very easy to determine that, like $\left(\Psi^{\mathrm{B}}\right)$ in Eq. $(3),(\psi)$ in Eq. $(6)$ is made out of one-forms. From Eqs. (28) and (29) of Ref. [5], the metric and vector potential variations are given by

$$
\left(\begin{array}{c}
\left(h_{\mu \nu}\right) \\
\left(b_{\mu}\right)
\end{array}\right) \equiv\left(\begin{array}{c}
\left(g_{\mu \nu}\right)^{\mathrm{B}} \\
\left(A_{\mu}\right)^{\mathrm{B}}
\end{array}\right)=\mathcal{S}^{\dagger}(\psi),
$$

where $\mathcal{S}^{\dagger}$ is an operator depending only on background fields (see Eqs. (26), (28), and (29) in Ref. [5]), which correspond to zero-forms. Since $h_{\mu \nu}$ and $b_{\mu}$ correspond to one-forms, from the preceding equation, $(\psi)$ correspond also to one-forms. This implies automatically that $J^{\mu}$ in Eq. (7) is a (nondegenerate) two-form on the corresponding phase space. However, we have to demonstrate that $J^{\mu}$ corresponds to a closed two-form for obtaining a symplectic structure on the phase space. For this purpose, we use the results in Appendix B. For example, the first term $-l^{\mu} M_{1^{\prime}} \wedge \tilde{\Psi}_{1}^{\mathrm{B}}$ for $J^{\mu}$ in Eq. (7) can be rewritten as:

$$
-l^{\mu} M_{1^{\prime}} \wedge \tilde{\Psi}_{1}^{\mathrm{B}}=\left[l^{\mu} M_{1^{\prime}}\left(\Psi_{1}-\frac{3 \Psi_{2}}{2 \phi_{1}} \phi_{0}\right)\right]^{\mathrm{B}},
$$

where Eq. (B1) has been used; the above expression means that such a term is an exact two-form (and automatically a closed two-form). Similarly, using Eqs. (B1) and (B2), the remaining terms in Eq. (7) correspond to closed two-forms. Therefore, $J^{\mu}$ itself is a closed two-form, as required for constructing a symplectic structure. Such geometrical structure is defined by $\omega \equiv \int_{\Sigma} J^{\mu} d \Sigma_{\mu}$, where $\Sigma$ is an initial value hypersurface. As $J^{\mu}$ is conserved, $\omega$ is independent of the choice of $\Sigma$ and, in particular, is Poincaré invariant [1]. In this manner, $\omega$ is a covariant symplectic structure on the phase space.

On the other hand, as shown in the Appendix A, the one-forms $\Psi_{0}^{\mathrm{B}}, \tilde{\Psi}_{1}^{\mathrm{B}}, \tilde{\kappa}^{\mathrm{B}}$, and $\tilde{\sigma}^{\mathrm{B}}$, are invariant under gauge transformations of the vector potential variations, $b_{\mu} \rightarrow b_{\mu}+\nabla_{\mu} \chi$, and thus, $J^{\mu}$ and $\omega$ have the same invariance property (in contrast with those of Refs. [1-4]). In field space, $\nabla_{\mu} \chi$ corresponds to the gauge directions, therefore, this invariance property defines automatically a gauge-invariant two-form $\omega$ on the subtler space $Z \equiv \hat{Z} / G$, being $\hat{Z}$ the space of solutions and $G$ the group of gauge transformations [1]. Similarly, since such one-forms are also invariant under infinitesimal rotations of the tetrad (see Appendix A), we have also a two-form $\omega$ independent on the perturbed tetrad gauge freedom, defined on the subtler space $T \equiv \hat{Z} / H$, being $H$ the group of infinitesimal rotations of the perturbed tetrad. However, $J^{\mu}$ and $\omega$ are not gauge invariant with respect to infinitesimal diffeomorphisms (in similarity to those of Refs. [1-4]). 
From Eq. (7) we can see that the conserved current depends on the background fields and the solutions admitted by the decoupled system and its adjoint. However, the entries of the matrix $\left(\Psi^{\mathrm{B}}\right)$ can be defined in terms of the metric and vector potential variations, which in turn, are defined in terms of the potential matrix $(\psi)$ (see Eqs. (28) and (29) of Ref. [5]), and therefore, $J^{\mu}$ and $\omega$ can be expressed in terms of a single complex solution of the equations for the potential $(\psi)$. However, in the more general case, if $(\psi)_{1}$ is a solution admitted by the equations for the potentials, the matrix $\left(\Psi^{\mathrm{B}}\right)$ can be expressed in terms of a second solution $(\psi)_{2}$, in general different of $(\psi)_{1}$. Therefore, $J^{\mu}$ and $\omega$ are expressed in terms of a pair of solutions for those equations. Finally, $J^{\mu}$ and $\omega$ will be given completely in terms of the potentials (one-forms), which become the fundamental geometrical structures. In this manner, the analysis of the structure of the phase space has been reduced to the study of equations for the potentials, which is a relatively simple issue.

Similarly, when the background electromagnetic field is null (and there is a possibly nonzero cosmological constant) the continuity equation for the field variations in the Einstein-Maxwell theory is given by (see Eqs. (21) and (24) of Ref. [6])

$$
\begin{aligned}
& \nabla_{\mu} J^{\mu}=0, \quad J^{\mu} \equiv l^{\mu}\left[\psi_{\mathrm{G}} \wedge(\Delta-4 \gamma+\mu) \Psi_{0}^{\mathrm{B}}+\psi_{\mathrm{E}} \wedge(\Delta-2 \gamma+\mu) \varphi_{0}^{\mathrm{B}}\right. \\
&\left.+2 \bar{\varphi}_{2}\left(\psi_{\mathrm{G}} \wedge(D-2 \epsilon) \varphi_{0}^{\mathrm{B}}-(D+4 \epsilon+3 \rho) \psi_{\mathrm{G}} \wedge \varphi_{0}^{\mathrm{B}}\right)\right] \\
&-n^{\mu}\left[(D+4 \epsilon+3 \rho) \psi_{\mathrm{G}} \wedge \Psi_{0}^{\mathrm{B}}+(D+2 \epsilon+\rho) \psi_{\mathrm{E}} \wedge \varphi_{0}^{\mathrm{B}}\right] \\
&-m^{\mu}\left[\psi_{\mathrm{G}} \wedge(\bar{\delta}-4 \alpha+\pi) \Psi_{0}^{\mathrm{B}}+\psi_{\mathrm{E}} \wedge(\bar{\delta}-2 \alpha+\pi) \varphi_{0}^{\mathrm{B}}\right] \\
&+ \bar{m}^{\mu}\left[(\delta+4 \beta+3 \tau) \psi_{\mathrm{G}} \wedge \Psi_{0}^{\mathrm{B}}+(\delta+2 \beta+\tau) \psi_{\mathrm{E}} \wedge \varphi_{0}^{\mathrm{B}}\right],
\end{aligned}
$$

where similarly $J^{\mu}$ is a closed two-form on the phase space, and the corresponding symplectic structure $\omega \equiv \int_{\Sigma} J^{\mu} d \Sigma_{\mu}$ is Poncairé invariant because $J^{\mu}$ is conserved. Furthermore, $J^{\mu}$ and the corresponding symplectic form $\omega$ have essentially the same invariance properties that the previous case (see Appendix A), and therefore are defined in a gauge-invariant form on the corresponding subtler spaces $Z$ and $T$. Analogously, they are given, in general, in terms of a pair of solutions admitted by the corresponding equations for the potentials (see Eqs. (24) in Ref. [6]).

In the case of solutions of the Einstein-Weyl equations such that the flux vector of the neutrino field is tangent to a shear-free congruence of null geodesics, the field variations satisfy the continuity 
equation (see Eqs. (38)-(47) of Ref. [包):

$$
\begin{array}{r}
\nabla_{\mu}\left\{l^{\mu}\left[-M_{1^{\prime}} \wedge \tilde{\Psi}_{1}^{\mathrm{B}}-\eta_{1} \psi_{\mathrm{G}} \wedge \tilde{\sigma}^{\mathrm{B}}-i k \eta_{1} \bar{\eta}_{1^{\prime}} M_{1^{\prime}} \wedge \tilde{\kappa}^{\mathrm{B}}+2 i k \eta_{1} \bar{\eta}_{1^{\prime}} M_{0^{\prime}} \wedge \tilde{\sigma}^{\mathrm{B}}\right]\right. \\
-n^{\mu}\left[M_{0^{\prime}} \wedge \Psi_{0}^{\mathrm{B}}+\eta_{1} \psi_{\mathrm{N}} \wedge \tilde{\kappa}^{\mathrm{B}}\right] \\
\left.+m^{\mu}\left[\eta_{1} \psi_{\mathrm{G}} \wedge \tilde{\kappa}^{\mathrm{B}}+M_{0^{\prime}} \wedge \tilde{\Psi}_{1}^{\mathrm{B}}-i k \eta_{1} \bar{\eta}_{1^{\prime}} M_{0^{\prime}} \wedge \tilde{\kappa}^{\mathrm{B}}\right]+\bar{m}^{\mu}\left[M_{1^{\prime}} \wedge \Psi_{0}^{\mathrm{B}}+\eta_{1} \psi_{\mathrm{N}} \wedge \tilde{\sigma}^{\mathrm{B}}\right]\right\}=0 .
\end{array}
$$

where $M_{0^{\prime}}, M_{1^{\prime}}, \psi_{N}$, and $\psi_{G}$ are the potentials, and similarly $\Psi_{0}^{\mathrm{B}}, \tilde{\Psi}_{1}^{\mathrm{B}}, \tilde{\sigma}^{\mathrm{B}}$, and $\tilde{\kappa}^{\mathrm{B}}$ are defined in terms of such potentials (see Eqs. (47) of Ref. [5).

The continuity equations (7)-(9) deserve some additional comments. The existence of such continuity laws follows directly from the definition of the adjoint of a differential operator, and we have only to identify conveniently the system of differential equations for applying such definition. In this sense, the adjoint operators scheme allows us to reveal the underlying geometrical structures for the corresponding theory, in a elementary and straightforward way. The argument involved is, as seen above, very simple.

On the other hand, in the Lagrangian approach known in the literature [2], the symplectic currents arise from the exterior derivative of the so called symplectic potentials, which correspond to one-forms on the phase space. This guarantees that such bilinear forms correspond automatically to closed two-forms (symplectic structures) on the phase space. However, in the present approach, the symplectic currents in Eqs. (7)-(9) are constructed in a direct way using only the concept of adjoint operators, regardless of the existence of symplectic potentials. Furthermore, our symplectic currents are generated from the usual derivatives (essentially directional derivatives) of the potentials, which correspond also to one-forms on the phase space. This suggests a possible relationship between the fundamental structures of the different approaches: the symplectic potentials of the Lagrangian approach and the potentials considered in the present scheme. This may be the subject of forthcoming works.

\section{CONCLUDING REMARKS}

As we have seen, the present approach based on the concept of the adjoint of a differential operator, 
represents a more direct and elementary procedure for constructing geometrical structures manifestly covariant on the phase space. Specifically, it has allowed us to demonstrate that, for the restricted class of spacetimes considered here, there exist two covariantly conserved currents for the field variations of each theory, the symplectic current (which coincides fully with that obtained from the Lagrangian approach [3, 由), and that found in the present approach (which is no obtained directly from a Lagrangian approach). Since both currents can be expressed, finally, in terms of solutions of the equations for the corresponding scalar potentials, our fundamental geometrical structures, a direct comparison is possible and convenient in order to establish the differences and similarities. On the other hand, although some restrictions on the background fields have been required, a lot of solutions of wide interest is contained in the cases considered here: rotating (charged) black holes, colliding plane waves, etc. The detailed study of the corresponding currents and geometrical structures associated with those particular solutions will be the subject of subsequent works. In fact, as mentioned in the Introduction, works along these lines have permitted to demonstrate that, the continuity equation (8) directly yields, in the particular case of the gravitational and electromagnetic perturbations of the Schwarzschild black hole, the conservation of the energy for those perturbations without invoking, as usually, the Schrödinger-type equation for establishing such a conservation relation. This result is connected with the existence of conserved quantities for non-Hermitian system of differential equations [7].

Finally, the general result established in Sec. II can be understood as an important extension of the original Wald's method [9]: wherever there exists an appropriate decoupled equation, it is not only possible to express the complete solution in terms of scalar potentials, but also to find automatically a corresponding (covariantly) conserved current, which can be expressed, in turn, in terms of the same potentials. The possible applications of such a result in the modern theories involving gravity and matter fields such as string theory (and other areas of physics) are open questions [7].

\section{ACKNOWLEDGMENTS}

This work was supported by CONACyT and the Sistema Nacional de Investigadores (México). 
The author wants to thank Dr. G. F. Torres del Castillo for his comments on the manuscript and suggestions, and Professor Robert Wald for the kind hospitality provided at the Enrico Fermi Institute, University of Chicago.

\section{APPENDIX A: INVARIANCE PROPERTIES}

Although it is not mentioned explicitly in Refs. [5, 5 , the perturbed quantities appearing in the decoupled equations are invariant under infinitesimal rotations of the tetrad. For example, in the case of the perturbed quantity $\tilde{\kappa}^{\mathrm{B}}$ (see Eqs. (4)) made out of the perturbations of the spin coefficient $\kappa$ and of the component of electromagnetic field $\phi_{0}$, we have that:

$$
\begin{aligned}
\kappa^{\mathrm{B}} & \equiv-\left(l^{\mu} l^{\nu} \nabla_{\nu} m_{\mu}\right)^{\mathrm{B}}=l^{\mu} l^{\nu} m_{\gamma}\left(\Gamma_{\mu \nu}^{\gamma}\right)^{\mathrm{B}}-l^{\mu} l^{\nu} \nabla_{\nu} m_{\mu}^{\mathrm{B}}-\left(l^{\mu} l^{\nu}\right)^{\mathrm{B}} \nabla_{\nu} m_{\mu} \\
& =l^{\mu} l^{\nu} m_{\gamma}\left(\Gamma_{\mu \nu}^{\gamma}\right)^{\mathrm{B}}-\frac{1}{2}(\bar{\pi}-\tau) l^{\mu} l^{\nu} h_{\mu \nu}-(D-2 \epsilon-\rho)\left(l^{\mu} m_{\mu}^{\mathrm{B}}\right),
\end{aligned}
$$

where $\left(\Gamma_{\mu \nu}^{\gamma}\right)^{\mathrm{B}}=\frac{1}{2} g^{\gamma \rho}\left(\nabla_{\mu} h_{\nu \rho}+\nabla_{\nu} h_{\mu \rho}-\nabla_{\rho} h_{\mu \nu}\right)$ and $h_{\mu \nu}$ is the metric perturbation. In this manner, the first and second terms in the above equation are defined completely in terms of $h_{\mu \nu}$. On the other hand, $l^{\mu} m_{\mu}^{\mathrm{B}}$ is dependent on the perturbed tetrad gauge freedom. Furthermore, from the definition $\phi_{0} \equiv l^{\mu} m^{\nu} F_{\mu \nu}$, one finds that

$$
\phi_{0}^{\mathrm{B}}=l^{\mu} m^{\nu} F_{\mu \nu}^{\mathrm{B}}-\left(\phi_{1}+\overline{\phi_{1}}\right) l^{\mu} m^{\nu} h_{\mu \nu}+2 \phi_{1}\left(l^{\mu} m_{\mu}^{\mathrm{B}}\right),
$$

where $\phi_{1}$ is the only nonvanishing component of the background electromagnetic field, and $F_{\mu \nu}^{\mathrm{B}}=$ $\partial_{\mu} b_{\nu}-\partial_{\nu} b_{\mu}$, being $b_{\mu}$ the vector potential perturbations. Therefore, from Eqs. (A1) and (A2) we can see easily that the quantity $\tilde{\kappa}^{\mathrm{B}}=\kappa^{\mathrm{B}}+(D-2 \epsilon-\rho)\left(\phi_{0}^{\mathrm{B}} / 2 \phi_{1}\right)$ is independent of the perturbed tetrad gauge freedom. Similarly for the remainding quantities $\Psi_{0}^{\mathrm{B}}, \tilde{\Psi}_{1}^{\mathrm{B}}$, and $\tilde{\sigma}^{\mathrm{B}}$ in Eq. (4). Thus, $\left(\Psi^{\mathrm{B}}\right)$ is invariant under the transformation considered. Since the field perturbation $F_{\mu \nu}^{\mathrm{B}}$ is invariant under the ordinary gauge transformation $b_{\mu} \rightarrow b_{\mu}+\nabla_{\mu} \chi$, where $\chi$ is an arbitrary scalar field, $\phi_{0}^{\mathrm{B}}$ in Eq. (A2) is also invariant under this transformation and, in this manner, $\left(\Psi^{\mathrm{B}}\right)$ is (see Eqs. (4)). However, it is not difficult to demonstrate that $\left(\Psi^{\mathrm{B}}\right)$ is not invariant under infinitesimal diffeomorphisms 
$b_{\mu} \rightarrow b_{\mu}+\mathcal{L}_{\chi} A_{\mu}$, and $h_{\mu \nu} \rightarrow h_{\mu \nu}+\mathcal{L}_{\chi} g_{\mu \nu}$, where $\chi^{\mu}$ is an arbitrary vector field, and $A_{\mu}$ and $g_{\mu \nu}$ the background fields.

Similarly, in the case of solutions of the Einstein-Maxwell theory with a null background electromagnetic field ( $\phi_{2}$ is the only nonvanishing component) [6], we find that

$$
\phi_{0}^{\mathrm{B}}=l^{\mu} m^{\nu} F_{\mu \nu}^{\mathrm{B}}+\frac{1}{2} \bar{\phi}_{2} l^{\mu} m^{\nu} h_{\mu \nu}
$$

which is independent of the perturbed tetrad gauge freedom and, of course invariant under $b_{\mu} \rightarrow$ $b_{\mu}+\nabla_{\mu} \chi$; however it is not under infinitesimal diffeomorphisms. On the other hand, it is easy to verify that $\Psi_{0}^{\mathrm{B}}$ is invariant under ordinary gauge transformations and infinitesimal diffeomorphisms (and independent of the perturbed tetrad gauge freedom). In this manner, our quantities are, in general, only invariant under both ordinary gauge transformations of the vector potential perturbartions and rotations of the tetrad.

\section{APPENDIX B: EXACT ONE-FORMS ON THE PHASE SPACE}

The field variations appearing in the decoupled equation (3) correspond to exact one-forms on the phase space.

The case of $\Psi_{0}^{\mathrm{B}}$ is trivial: $\Psi_{0}^{\mathrm{B}}$ is directly the exterior derivative of the zero-form $\Psi_{0}$ (see last paragraph in Sec. II).

The other cases are more elaborated. For example, $\tilde{\Psi}_{1}^{\mathrm{B}}$ can be rewritten as:

$$
\tilde{\Psi}_{1}^{\mathrm{B}} \equiv \Psi_{1}^{\mathrm{B}}-\frac{3 \Psi_{2}}{2 \phi_{1}} \phi_{0}^{\mathrm{B}}=\Psi_{1}^{\mathrm{B}}-\left(\frac{3 \Psi_{2}}{2 \phi_{1}} \phi_{0}\right)^{\mathrm{B}},
$$

since $\phi_{0}=0$ in the background. Similarly, $\tilde{\kappa}^{\mathrm{B}}$, and $\tilde{\sigma}^{\mathrm{B}}$ can be rewritten as:

$$
\begin{aligned}
& \tilde{\kappa}^{\mathrm{B}}=\kappa^{\mathrm{B}}+\left[(D-2 \epsilon-\rho) \frac{\phi_{0}}{2 \phi_{1}}\right]^{\mathrm{B}}, \\
& \tilde{\sigma}^{\mathrm{B}}=\sigma^{\mathrm{B}}+\left[(\delta-2 \beta-\tau) \frac{\phi_{0}}{2 \phi_{1}}\right]^{\mathrm{B}} .
\end{aligned}
$$


Therefore, from Eqs. (B1) and (B2), $\tilde{\Psi}_{1}^{\mathrm{B}}, \tilde{\kappa}^{\mathrm{B}}$, and $\tilde{\sigma}^{\mathrm{B}}$ correspond to the exterior derivative of background zero-forms, and then correspond to exact one-forms. A consequence of this property is that $\left(\Psi^{\mathrm{B}}\right)$ is made out of closed one-forms:

$$
\left(\Psi^{\mathrm{B}}\right)^{\mathrm{B}}=0 .
$$

Similarly, the field variations $\Psi_{0}^{\mathrm{B}}, \tilde{\Psi}_{1}^{\mathrm{B}}, \tilde{\kappa}^{\mathrm{B}}$, and $\tilde{\sigma}^{\mathrm{B}}$ appearing in Eq. (9) (and defined in Eqs. (39) of Ref. [5]), correspond to exact one-forms on the phase space.

The cases of $\Psi_{0}^{\mathrm{B}}$ and $\phi_{0}^{\mathrm{B}}$ in Eq. (8) are also trivials: they are directly the variations of the corresponding background quantity.

\section{References}

[1] C. Crncović and E. Witten, in Three Hundred Years of Gravitation, edited by S. W. Hawking and W. Israel (Cambridge, University Press. Cambridge, 1987).

[2] J. Lee and R. M. Wald, J. Math. Phys. 31, 3 (1990); G. A. Burnett and R. M. Wald, Proc. R. Soc. London A430, 57 (1990).

[3] R. Cartas-Fuentevilla, Phys. Rev. D 57, 3443 (1998).

[4] G. F. Torres del Castillo and J. C. Flores-Urbina, Gen. Rel. Grav. 31, 1315 (1999).

[5] G. F. Torres del Castillo, J. Math. Phys. 5, 649 (1988).

[6] G. F. Torres del Castillo, J. Math. Phys. 37, 4053 (1996).

[7] R. Cartas-Fuentevilla, J. Math. Phys. 41, 7521 (2000).

[8] G. F. Torres del Castillo, Gen. Rel. Grav. 22, 1085 (1990).

[9] R. M. Wald, Phys. Rev. Lett. 41, 203 (1978). 